\title{
Distinct patterns of MMP-9 and MMP-2 activity in slow and fast twitch skeletal muscle regeneration in vivo
}

\author{
MALGORZATA ZIMOWSKA* , EDYTA BRZOSKA, MARTA SWIERCZYNSKA, WLADYSLAWA STREMINSKA \\ and JERZY MORACZEWSKI \\ Department of Cytology, Institute of Zoology, Faculty of Biology, University of Warsaw, Warsaw, Poland
}

\begin{abstract}
Skeletal muscles exhibit great plasticity and an ability to reconstruct in response to injury. However, the repair process is often inefficient and hindered by the development of fibrosis. We explored the possibility that during muscle repair, the different regeneration ability of the fast (extensor digitorum longus; EDL) and slow twitch (Soleus) muscles depends on the differential expression of metalloproteinases (MMP-9 and MMP-2) involved in the remodeling of the extracellular matrix. Our results show that MMP-9 and MMP-2 are present in the intact muscle and are up-regulated after crush-induced muscle injury. The expression and the activity of these two enzymes depend on the type of muscle and the phase of muscle regeneration. In the regenerating Soleus muscle, elevated levels of MMP-9 occurred during the myolysis and reconstruction phase. In contrast, regenerating EDL muscles exhibited decreased MMP-9 levels during myolysis and increased MMP-2 activity at the reconstruction phase. Moreover, satellite cells (mononuclear myoblasts) derived from Soleus and EDL muscles showed no differences in localization or activity of MMP-9 and MMP-2 during proliferation and differentiation in vitro. MMP9 activity was present during all stages of myoblast differentiation, whereas MMP-2 activity reached its highest level during myoblast fusion. We conclude that MMPs are involved in muscle repair, and that fast and slow twitch muscles exhibit different patterns of MMP-9 and MMP-2 activity.
\end{abstract}

KEY WORDS: MMP-9, MMP-2, skeletal muscle regeneration, myogenesis in vitro

\section{Introduction}

Skeletal muscle has a great plasticity and unique ability to reconstruct in response to injury (Allbrook, 1981, Chambers and McDermott, 1996, Schultz, 1989). The regeneration occurs in two overlapping stages: myolysis and reconstruction. Myolysis phase encompasses active muscle degeneration and inflammation processes that occur in the first few days post-injury. Numerous macrophages and leukocytes gather at the site of injury and phagocyte fragments of necrotic cells (Teixeira etal., 2003, Tidball, 2005). The reconstruction of muscle relies on the pool of tissue specific, undifferentiated myogenic precursor cells, known as satellite cells (mononuclear myoblasts) (Mauro, 1961). In the intact muscle, satellite cells remain mitotically quiescent, but become activated in response to muscle injury, denervation, stretching or exercise. Upon activation, the satellite cells resume proliferation. After several cell cycles the majority of these cells starts to differentiate and fuse to form new myofibers or to repair damaged myofibers (Anderson, 1998, Charge and Rudnicki, 2004, Schultz, 1989).

Mature muscle is a mosaic of fast and slow twitch fibers (Huard et al., 2002). Due to their fiber composition, the fast twitch muscle Extensor digitorum longus (EDL) containing about $95 \%$ of fast fibers, and the slow twitch muscle Soleus containing $80-100 \%$ of slow fibers, are widely used as the models of fast and slow muscles. It has been shown that there are differences in the regenerative response of these two muscle types to microlesion injury (Darr and Schultz, 1987), injection of anesthetic (Kalhovde et al., 2005, Nonaka et al., 1983) or crush (Bassaglia and Gautron, 1995). In crush induced regeneration model, EDL muscle regenerates properly and shows a normal structure at day 14 after wounding (Bassaglia and Gautron, 1995). In contrast, Soleus muscle undergoes fibrosis after crush and denervation (Bassaglia

Abbreviations used in this paper: ECM, extracellular matrix; EDL, extensor digitorum longus muscle; MMP, matrix metalloproteinase.

\footnotetext{
*Address correspondence to: Malgorzata Zimowska. Department of Cytology, Institute of Zoology, Faculty of Biology, University of Warsaw, 1 Miecznikowa St., 02-096 Warsaw, Poland. e-mail: mzimowska@biol.uw.edu.pl
} 
and Gautron, 1995, Moraczewski et al., 1996, Zimowska et al., 2001).

Muscle repair is a finely orchestrated process involving changes in the synthesis and degradation of the components of ECM, commonly described as ECM remodeling (Charge and Rudnicki, 2004). Previous reports have indicated that the zinc-dependent matrix metalloproteinases (MMPs) play the pivotal role in ECM remodeling during muscle regeneration (Tsuruda et al., 2004, Werb and Chin, 1998). MMPs family includes more then 20 identified members which digest the ECM components (McCawley and Matrisian, 2001, Ravanti and Kahari, 2000). It has been shown that two isoforms of metalloproteinases - MMP-2 and MMP-9 (gelatinase $\mathrm{A}$ and $\mathrm{B}$, respectively) - are up-regulated in muscle undergoing regeneration after injection with cardiotoxin (Kherif et al., 1999). Activation of MMP-2 and MMP-9 has also been found to be associated with various myopathic and inflammatory-induced changes in skeletal muscle (Kieseier et al., 2001, Schoser et al., 2002). Moreover, in $m d x$ murine muscles, that serve as animal model of Duchenne dystrophy, the constitutive expression of MMP-9 and the up-regulation of MMP-2 have been observed (Kherif et al., 1999).

Since muscle crush results in structural rebuilding of muscle accompanied by ECM remodeling, we investigated the differences in MMP-9 and MMP-2 activity between regenerating Soleus and EDL muscles. We hypothesized that in contrast to properly regenerating EDL muscle, extensive fibrosis found in the regenerating Soleus muscle may be linked with changes in MMP-9 and/or MMP2 activity. Thus, the aim of our study was to establish if there are differences between fast and slow muscles in MMP-9 and MMP-2 activity, expression and localization during the regeneration process.

Effective muscle regeneration requires a coordinated repair response involving satellite cells, inflammatory cells and vascular system to restore the architecture of the tissue. Since previous studies have demonstrated that murine myoblast can produce both MMP-2 and MMP-9 (Allen et al., 2003, El Fahime et al., 2000, Ohtake et al., 2006), we analyzed the contribution of myoblasts isolated from Soleus and EDL muscles to MMP-9 and MMP-2 production during slow and fast twitch muscles repair. In the present study we determined the levels of MMP-9 and MMP-2 activity and expression at protein and mRNA levels and the localization of both enzymes during myoblast differentiation in vitro. Therefore, we were able to distinguish whether the morphological differences observed during regeneration of Soleus and EDL muscles result from the activity of their satellite cells or the inflammatory cells, which infiltrate crushed areas.

\section{Results}

\section{MMP-9 and MMP-2 are simularly distributed during Soleus and EDL muscle regeneration}

Using crush induced muscle regeneration model we investigated repair process of slow twitch Soleus (Fig. 1A) and fast twitch EDL muscles (Fig. 1C). In this experimental model, muscles underwent complete degeneration within the first 3 days after the procedure. Then, the process of reconstruction of muscle fibers started. In contrast to EDL muscle, that regenerated properly and showed a well-structured muscle at day 14 after crush, Soleus muscle displayed heterogeneous tissue repair characterized by the accumulation of ECM components (Fig. 1A). Since metalloproteinases are believed to play a key role in the degradation of ECM components, first we investigated localization of MMP9 and MMP-2 during muscle regeneration.

In the intact Soleus (Fig. 1B) and EDL muscles (Fig. 1D) both metalloproteinases were detected around muscle fibers. At day 1 of the regeneration, when the pronounced myolysis was observed, MMP-9 and MMP-2 were localized around destroyed muscle fibers and decorated cells localized between them. These cells were presumably inflammatory cells or activated satellite cells. During the repair process MMP-2 and MMP-9 were found in the cytoplasm of young myofibers and mononuclear cells. At day 14 after crush the regression of inflammatory edema occurred and MMP-2 and MMP-9 were found mostly in the cytoplasm and around the young myofibers. The distribution of MMP-9 and MMP-2 was similar in
Fig. 1. Immunolocalization of MMP-9 and MMP-2 during muscle regeneration. $(A, B)$ Soleus muscle; $(C, D)$ EDL muscle. Histological aspects of the regeneration of Soleus (A) and EDL (C) muscles. The injured muscles underwent complete degeneration within the first three days post injury. The inflammatory cells invaded the injured muscles at day 1 after injury. By day 7 , numerous young myotubes regenerated and inflammation decreased. Maturation of myotubes into multinucleated myofibers progressively occurred and by the day 14 EDL muscle was properly regenerated. In contrast, during the same time Soleus muscle underwent fibrosis. Observations were performed using a Nikon microscope. Immunolocalization of MMP-9 and MMP-2 during regeneration of Soleus (B) and EDL (D) muscles. Immunostaining for MMP-9 and MMP-2 (green) were carried out. The images were recorded using a LSM 510 confocal system (Zeiss).
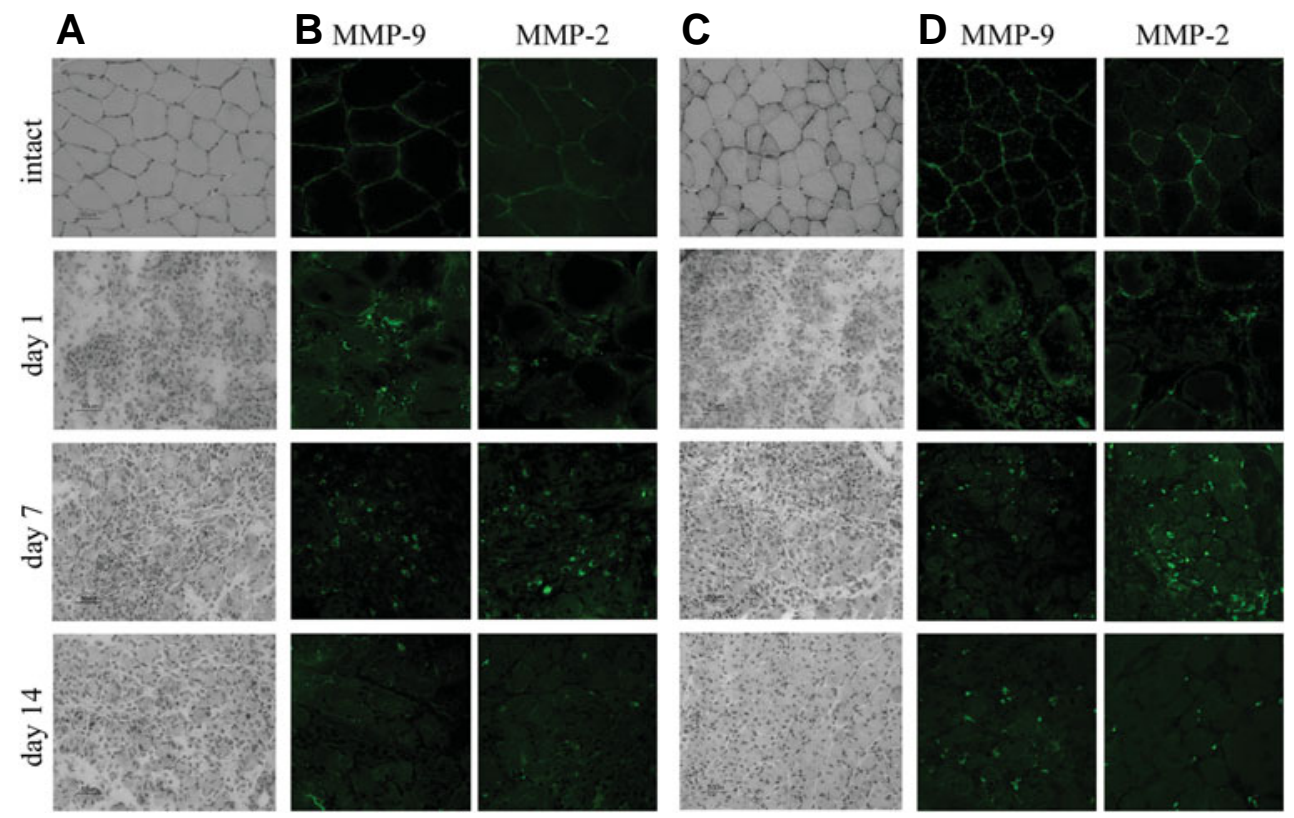
Soleus and EDL muscles at different phases of muscle repair.

\section{MMP-9 and MMP-2 mRNA levels change during Soleus and EDL regeneration}

Although no differences in distribution of MMP-9 and MMP-2 were observed during regeneration of Soleus and EDL muscles, we decided to compare levels of mRNAs encoding MMP-2 and MMP-9 in both muscles at different stages of regeneration (at day 0 and 3,5,7 and 14).

MMPs expression showed differences in Soleus and EDL muscles (Fig. 2A). Analysis of MMP-9 mRNA revealed its highest expression in the injured muscles and at the myolysis phase (day 3 ) in both Soleus and EDL muscles. The level of MMP-9 mRNA was also high at the early reconstruction phase in EDL (day 5) but it decreased at day 7. No MMP-9 mRNA was found in the regenerated EDL muscle (day 14). In contrast, in Soleus muscle MMP-9 mRNA level decreased at day 5 of regeneration and remained at the same level up to day 14.

MMP-2 mRNA was present at low level in the injured muscles and at the myolysis phase. However, the level of MMP-2 mRNA was higher in Soleus than in EDL muscle. It increased at the reconstruction phase reaching the highest level at day 14 in Soleus and at days 5 and 7 in EDL muscle. Therefore EDL and Soleus muscles show different expression of MMPs mRNA depending on the stage of regeneration process.

\section{Levels of MMP-9 and MMP-2 protein change during muscle regeneration}

We performed Western blot analyses of both metalloproteinases in protein extracts prepared from the regenerating muscles at different time points (day 1, 3, 5, 7 and 14 after crush) (Fig. 2B). At day 1 of regeneration, amount of MMP-9 was about 10 times higher in Soleus and 5 times higher in EDL muscle than in the intact muscles (Fig. 2B). In Soleus muscle the level of MMP9 protein gradually decreased up to day 14 after crush but remained about 5 times higher than in the intact muscle. The level of MMP-9 found in the regenerating EDL muscle gradually decreased reaching the level found in the intact muscle at day 14 after crush. Level of MMP-9 protein found in Soleus muscle was higher than that in EDL muscle at every stage of regeneration.

MMP-2 protein level highly increased at day 1 of regeneration in Soleus muscle and remained high up to day 14 after crush. In contrast, in EDL muscle only a slight increase in the amount of MMP-2 was observed at 1 day of regeneration compared with the intact muscle. However, during the next days of regeneration the level of MMP-2 continuously increased reaching the peak at day 7 after the crush. At this time point the level of MMP-2 was about 10 times higher in the regenerating EDL than in the intact muscle. Then, in the regenerated EDL muscle the amount of MMP. 2 protein decreased. Nevertheless, it remained higher than in the intact muscle. Thus, EDL and Soleus muscles show different patterns of MMP-2 and MMP9 protein expression at different stages of regeneration.

\section{MMP-9 and MMP-2 exhibit different activity profiles during regeneration of Soleus and EDL muscles}

The differences in mRNA levels of MMP-2 and MMP-9 between regenerating Soleus and EDL muscles were accompanied by the changes in the levels of proteins of both enzymes (described above). We wanted to determine whether these observations correlate with the differences in the activity of both metalloproteinases. Since MMP-9 and MMP-2 are known to be gelatinases, their activity was determined by gelatin-substrate zymography under nonreducing condition (Fig. 2C). In the intact muscles both MMP-9 and MMP-2 activity was low. MMP-9 activity increased at day 1 after crush in both Soleus and EDL muscles. However, its activity was about 2 times lower in EDL when compared with Soleus muscle. In the regenerating Soleus MMP9 activity continuously decreased until day 14 after crush but at every stage of regeneration it was at least 5 times higher than in the intact control muscle. In the regenerating EDL muscle activity of MMP- 9 remained high up to day 5 after crush but at day 7 it decreased to the level observed in the intact control muscle. It remained at this level up to day 14 after crush.

MMP-2 also exhibited different activity profiles in the extracts from the regenerating Soleus and EDL muscles. During Soleus muscle regeneration the low activity of MMP-2, comparable to that found in the intact control muscle, was observed during myolysis (from 1 to 3 days after crush). From day 5 after crush it gradually increased reaching the highest level at day 14. In contrast, in the regenerating EDL muscle the high activity of MMP-2 occurred at day 1 . Then it increased reaching the maximum at day 7 after crush and remained high until day 14 .

\section{MMP-9 and MMP-2 show different localization in differentiat- ing myoblasts}

The immunostaining of muscles during repair showed labeling

\section{MMP-9}

MMP-2

soleus
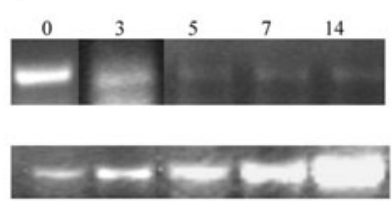

MMP-9

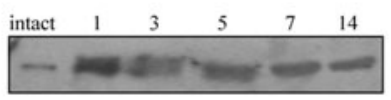

MMP-2

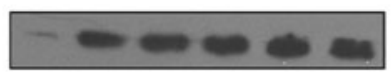

MMP-9

MMP-2

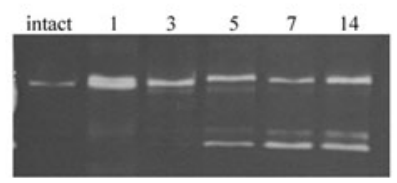

A
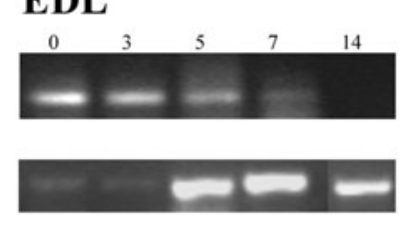

B
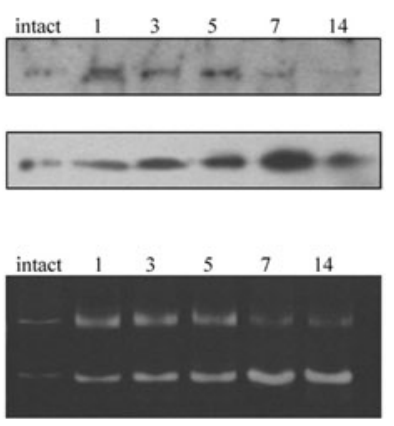

Fig. 2. Characterization of MMP-9 and MMP-2 during Soleus and EDL muscle regeneration. Samples of the regenerating or intact muscles were prepared as describes in Mat. and Met. and loaded per lane. (A) RT-PCR assay. (B) Western blott analysis. (C) Zymographyanalysis. Gels and blots were documented using Gel Doc 2000 scanner. 
of mononuclear cells suggesting that MMPs found in the regenerating muscles might be attributable not only to the infiltrating inflammatory cells but also to the resident myogenic cells. Therefore, we investigated immunolocalization of MMP-9 and MMP-2 in differentiating myoblasts in vitro to verify the hypothesis that differences between fast and slow type muscles might result, at least in part, from intrinsic properties of myoblasts.

During the first three days of cell culture in vitro, the myoblasts adhered to the plates and proliferated at a very low rate (Fig. $3 A, C$ ). In the next two days, the rate of myoblast divisions increased. Subsequently, the myoblasts aligned, adhered tightly and fused to form myotubes. In Soleus myoblast cultures the first myotubes appeared at day 7. At day 11, the cultures contained long, multinuclear myotubes. Two days later about $60 \%$ of myoblasts were fused. The differentiation of EDL derived myoblasts (Fig. 3C) was 1-2 days delayed in comparison to Soleus myoblasts (Fig. 3A).

The immunostaining showed no significant differences in MMP distribution between Soleus (Fig. 3B) and EDL (Fig. 3D) derived cells. MMP-2 and MMP-9 were observed at the membrane of satellite cells fixed and processed immediately after the isolation from EDL and Soleus muscles. During the proliferation phase (days 3-5) MMP-9 was mostly localized in the cytoplasm of mononuclear cells and only a weak signal was observed at the myoblast membrane. When myoblasts fused with each other (days 7-9) MMP-9 was still visible within the cytoplasm, however signal at the membrane became stronger. In most multinuclear myotubes,
MMP-9 was evenly distributed in cytoplasm, however, MMP-9 was absent from some mature myotubes.

MMP-2 was present in myoblasts cytoplasm at all phases of differentiation. However, at the proliferation phase MMP-2 was also found at the plasma membrane of both Soleus and EDL derived myoblasts. MMP-2 localization along plasma membrane was especially conspicuous during fusion phase, when myoblasts were in close contact with each other or with other myotubes. Thus, localization of both enzymes was similar in myoblast isolated from Soleus and EDL muscles, but distribution of MMP-9 and MMP-2 was different at proliferation and differentiation phase suggesting different role of both enzymes in these processes.

\section{Differences in MMP-9 and MMP-2 mRNA expression during differentiation of myoblasts isolated from Soleus and EDL in vitro}

Since the distribution of MMP-9 and MMP-2 in Soleus and EDL derived myoblasts varied depending on the phase of differentiation we decided to compare levels of mRNA encoding these enzymes in myoblasts derived from both muscles at different phases of their differentiation (Fig. 4A).

Samples were obtained from satellite cells isolated directly from the muscles (day 0) and myoblasts from day 5, 7 and 11 of culture.

MMP-9 mRNA was present in Soleus and EDL derived myoblasts. In Soleus derived myoblasts MMP-9 mRNA remained at the
A

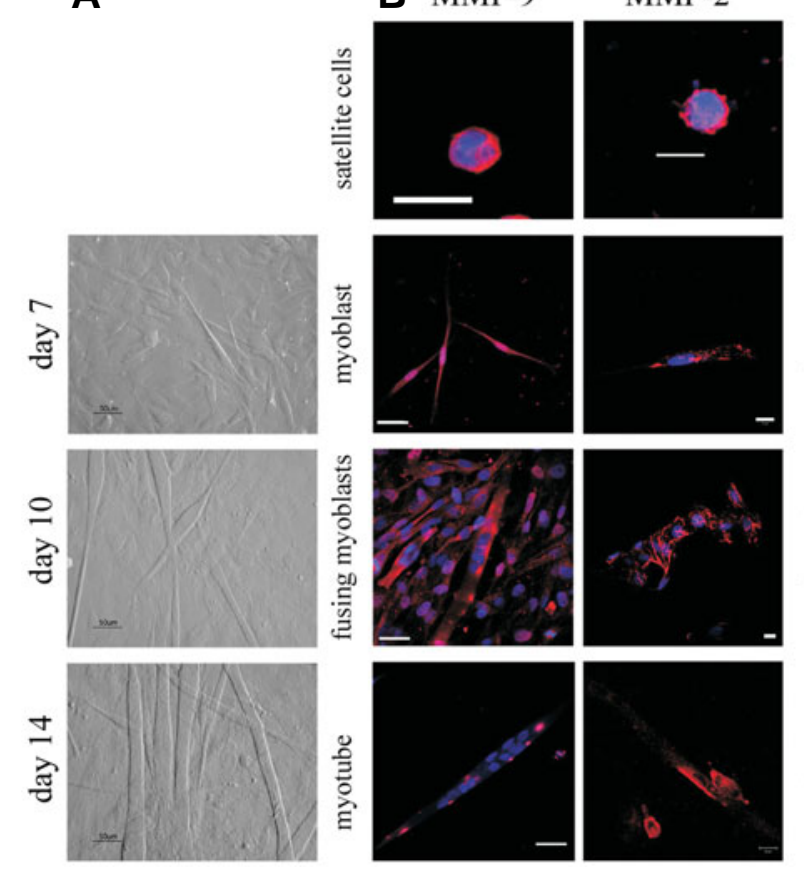

C

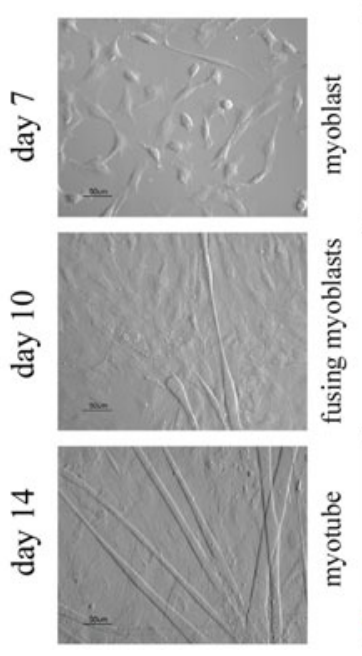

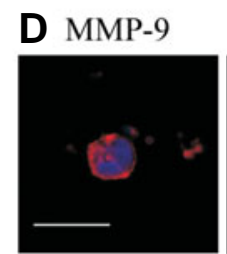

MMP-2
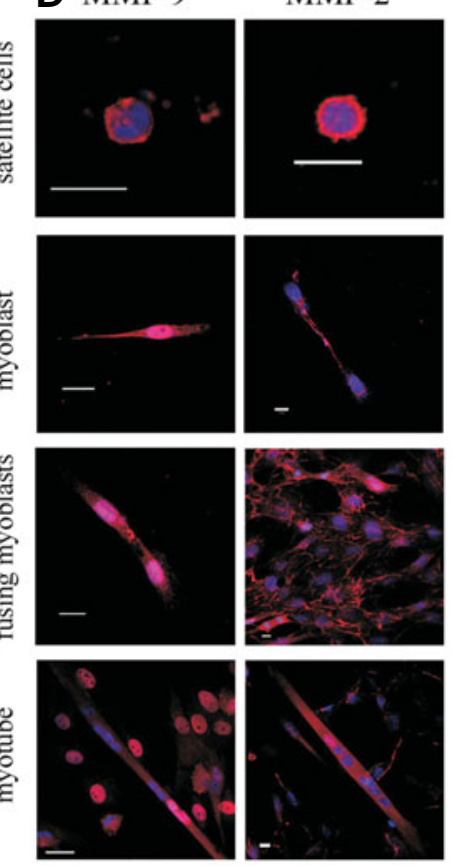

Fig. 3. Immunolocalization of MMP-9 and MMP-2 during myoblast differentiation. (A, B) Soleus muscle. (C,D) EDL muscle. Morphological aspects of cultures of myoblasts isolated from Soleus (A) and EDL (C) muscles. Differentiation of myoblasts was induced in DMEM containing $10 \%$ FBS and $10 \%$ horse serum. Myoblasts adhered to plates, proliferated and then aligned and fused with one another. In Soleus myoblast cultures, the first myotubes appeared at day 7. Differentiation of EDL derived myoblasts was delayed and the first myotubes were formed at day 10 in vitro. By day 14, $60 \%$ of myoblasts underwent fusion in both types of cultures. Myotubes found in Soleus satellite cell cultures were bigger and contained more nuclei compared with EDL ones. Histological aspects of cultures were assessed by Hoffman contrast observation (Nikon microscope). Immunolocalization of MMP-9 or MMP-2 during differentiation of myoblasts isolated from Soleus (B) and EDL (D) muscles. Myoblasts at different phases of growth were fixed with 3\% paraformaldehyde in PBS and stained with antibodies against MMP-9 and MMP-2 (red) and chromomycin (blue). The images were recorded using a LSM 510 confocal system (Zeiss). 
same low level during all phases of myoblast differentiation. In contrast, in EDL derived myoblast high expression of MMP-9 mRNA was observed in isolated satellite cells (day 0 ) and in proliferating myoblasts (day 5) but it decreased at the beginning of myoblasts fusion (days 7 and 11).

MMP-2 mRNA was highly expressed in satellite cells isolated from Soleus muscle and remained high in proliferating, fusing (day 7) myoblasts and in myotubes at day 11 of culture. In contrast, the level of MMP-2 mRNA was low in satellite cells and proliferating myoblasts derived from EDL muscle but it increased in fusing myoblasts and in the mature myotubes. Therefore expression of MMPs mRNA in satellite cells and cultured myoblasts differed depending on the muscle cells origin and the stage of their differentiation.

\section{Distinct levels of MMP-9 and MMP-2 protein during myoblast differentiation}

Western blotting analysis of MMP-9 and MMP-2 in Soleus and EDL derived myoblasts was performed from 4 to 14 days cultures (Fig. 4B). MMP-9 protein levels increased at proliferation phase. The highest level of MMP-9 was found at day 6 and day 8 in Soleus and EDL derived myoblasts, respectively. MMP-2 levels did not change markedly during cell proliferation and differentiation in Soleus derived myoblasts. However, in EDL derived myoblasts, at day 10 of culture, when fusion began, MMP-2 amount increased reaching the level about 3 times higher than the level found at proliferation phase. Thus, the myoblasts from Soleus and EDL muscles express MMP-9 and MMP-2 in vitro and display differential pattern of MMP-9 and MMP-2 expression during differentiation.

\section{MMP-9 and MMP-2 have different activity during myoblast pro- liferation and differentiation in vitro}

We analyzed MMP-2 and MMP-9 activity in medium conditioned by Soleus and EDL derived myoblasts cultured in vitro(Fig. 4C). The assay was performed using samples collected from 4 to 14 days cultures. In Soleus derived myoblasts MMP-9 activity increased during the proliferation phase reaching the highest level at day 6 of culture. At day 8 of culture, during myoblasts differentiation its activity decreased to the level found at day 4 and remained at the same level until day 14 of culture. The pattern of MMP-9 activity found in EDL derived myoblast culture was similar to that found in Soleus. MMP9 activity slightly increased at day 8 of culture in EDL. In contrast, in Soleus derived myoblasts MMP-2 activity gradually increased starting from day 6 and reached the highest level at day 10 of culture when the fusion occurred. In EDL-derived myoblasts activity of MMP-2 did not change markedly during proliferation phase (days 4-8) and increased about 3 times at day 10 after plating, reaching its highest level at day 12 when the fusion occurred. Thus activity of MMP-9 and MMP-2 is related to phase of myoblasts differentiation

\section{Discussion}

Several studies have shown that MMPs are involved in degradation of ECM components in muscle (Demestre et al., 2005, Haas et al., 2000). However fast and slow twitch muscles display number of soleus

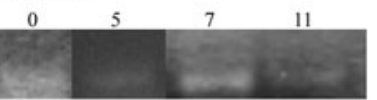

A

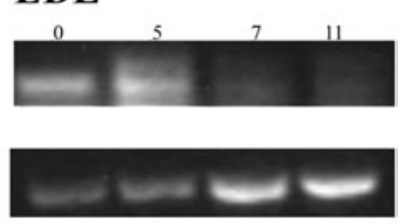

B
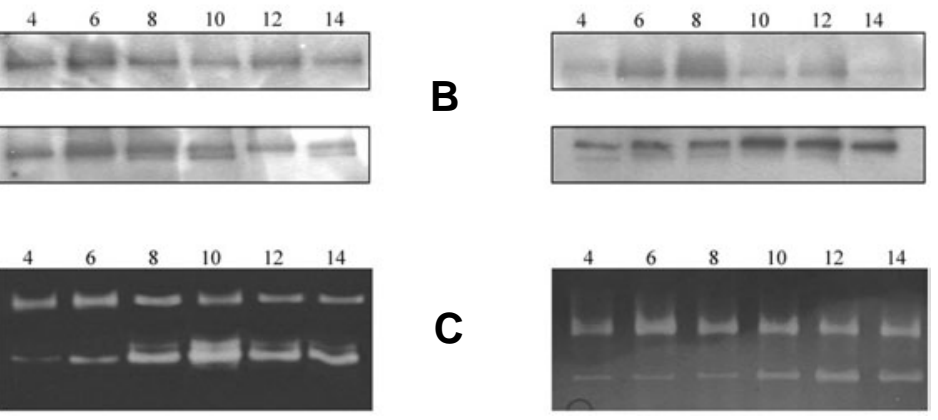

C

differences in the ECM composition. The main hypothesis exfast (EDL) and slow twitch (Soleus) muscles might result from differential MMPs action in ECM remodeling. In our current study we extended previous analyses of MMPs (Demestre et al., 2005) to the study of the localization of MMP-9 and MMP-2. Our results confirm that in the intact muscles only a weak immunostaining signal for MMP-9 and MMP-2 was detected around muscle fibers. After experimental crush injury we observed strong immunostaining along the sarcolemma of diseased muscle fibers, in mononuclear cells and along the sarcolemma and in the cytoplasm of newly formed myofibers. We found that the expression of MMP-9 and MMP-2 is up-regulated after the muscle injury. Nevertheless, the expression and activity of these two enzymes show different patterns depending on the type of muscle and the phase of muscle regeneration. The activity of MMP-9 seems to be more important at the beginning of muscle repair in Soleus and EDL. However, MMP-9 activity remains higher for longer period (up to day 14 post injury) in poorly regenerating Soleus muscle while in EDL muscle, at day 7 after crush, the MMP-9 activity decreased to the level observed in the intact control muscle.

Injury initiates a rapid and sequential invasion of muscle by inflammatory cells - neutrophiles followed by macrophages (Tidball, 2005). The invading inflammatory cells have the ability to release MMPs (Schoser et al., 2002). On the other hand, it has been demonstrated that MMP-9 is up-regulated during nerve crush whereas its level decreases when the muscle becomes reinervated (Demestre et al., 2005). In our crush induced regeneration model, Soleus myolysis was rapid, extensive and heterogeneous and accompanied by increase in blood flow when compared to EDL muscle. On the other hand Bassaglia and Gautron (1995) showed that the reinervation of EDL muscle was faster than Soleus muscle (Bassaglia and Gautron, 1995). This may suggest that MMP-9 is 
secreted mainly by non muscle cells including inflammatory cells, and different patterns of MMP-9 activity found in slow and twitch muscle may reflect differences in their response to injury at the myolysis phase and at the beginning of reinervation.

MMP-2 activity that increases gradually during muscle regeneration seems to be more important at the reconstruction phase. Interestingly, the increase in MMP-2 protein level (demonstrated by Western blotting) in the regenerating Soleus muscle was accompanied by low MMP-2 enzymatic activity at the beginning of muscle repair. In contrast, in the regenerating EDL muscle the increased MMP-2 protein level was accompanied by its high enzymatic activity. Regulation of MMP-9 and MMP-2 enzymatic activity is a complex process. It can be regulated at the level of synthesis, zymogen activation and control of enzyme activity by tissue inhibitors of metalloproteinases (TIMP) (Kugler, 1999). Thus the differences in MMP-2 enzymatic activity and differences in MMP-2 protein level found in Soleus and EDL muscles may represent different mechanism of activity control.

Our data suggest that the different pattern of MMPs activity in slow and fast twitch muscles may be due to the different muscle environments. Crush induced regeneration model, used in our studies, showed that fast twitch muscle EDL had higher activity of MMP-2 than slow twitch Soleus muscle. The high activity of MMP-2 found in EDL muscle seems to be responsible for $\mathrm{ECM}$ remodeling during reconstruction phase and prevent the accumulation of ECM components. Thus the extensive fibrosis evident in Soleus muscle could result, at least partially, from the insufficient degradation of ECM components.

The muscle environment has a profound effect on the regenerative capacity of the resident muscle precursor cells (Partridge, 2006). Primary cultures of myoblasts isolated from Soleus and EDL muscles allowed to determine whether differences in MMPs activity during repair process result from intrinsic properties of myoblasts. There are many contradictory reports concerning the role of MMPs in myoblasts differentiation. It has been shown that overexpression of MMP-2 in culture of mouse myoblasts significantly augments myoblasts migration, whereas MMP-9 seems to play a minimal role in this process (Allen et al., 2003). It has been also postulated that regulation of matrix turnover via MMP-9 may be involved in the events leading to myotubes formation (Lewis et al., 2000). Our results indicate that MMP-9 is involved in all stages of myoblasts differentiation, whereas MMP-2 seems to play a crucial role during cell fusion. Immunolabeling of MMP-2 found at the plasma membrane in fusing myoblasts seems to confirm the specific role of this enzyme during cell fusion.

Our results do not indicate a direct causal relationship between satellite cells ability to produce MMPs and regeneration ability of slow and fast twitch muscles. We found no striking differences between Soleus and EDL derived cells in localization and activity of MMP-9 and MMP-2 during myoblasts proliferation and differentiation. Thus the contribution of myoblasts in vivo to the production of MMPs and remodeling of muscle ECM might be lower than the contribution of other cell types including fibroblasts, inflammatory and endothelial cells. However, since the muscle environment has a profound effect on the regenerative capacity of resident muscle precursor cells, the differences of MMP-9 and MMP-2 activity, found in Soleus and EDL regenerating muscles may modify myoblasts migration and fusion and be essential for the differences in the regenerative response of both muscle types.

\section{Materials and Methods}

\section{Regeneration}

The regeneration of EDL and Soleus muscles was induced in adult white Wag male rats as previously described [Bassaglia and Gautron, 1995]. All procedures involving animals were approved by Local Ethics Committee no 1 in Warsaw. Rats were anaesthetized with pentobarbital (Serva) and the muscle was exposed and then denervated by cutting the motor nerve at the entrance of the muscle. Next the muscle region located between two tendons was crushed with serrated jaws. After skin closure animals were allowed to recover and were returned to their cages. At different days after the procedure (regeneration days $1,3,5,7$ and 14) animals were euthanatized using $\mathrm{CO}_{2}$ (Linde Gas) and the regenerating muscles were removed and weighed. Muscles were then either directly used for biochemical investigations or froze in isopentane (Fluka) pre-cooled in liquid nitrogen, and then stored at $80^{\circ} \mathrm{C}$ for further histological analysis. Transverse $10 \mu \mathrm{m}$ thick sections of Soleus and EDL muscles were obtained by cryosectioning and then stained with the Gomori Trichrome (Sigma) technique for histological examination. At each day of regeneration, 3 rats were used for the examination of each muscle. Experiments were repeated three times.

\section{Myoblast primary cultures}

Satellite cells were dissociated with pronase (Sigma) from EDL or Soleus muscles isolated from 3 month old Wag male rats, as previously described (Lagord et al., 1998). Myoblasts were seeded (20 000 cells/ $\mathrm{cm}^{2}$ ) on slides covered with adhesion promoting molecules (Poly-LLysine, Sigma). For Western blotting or zymography cells were seeded on dishes coated with $0.1 \%$ gelatin (Sigma) and grown continuously in DMEM (Gibco) containing 10\% fetal bovine serum (FBS) and 10\% horse serum (HS) (Gibco) in $5 \% \mathrm{CO}_{2}$ at $37^{\circ} \mathrm{C}$. At days $4,6,8,10,12$ or 14 medium from the cultures was collected for zymography and cells were homogenized for Western blotting. For an immunocytological analysis Soleus and EDL derived myoblasts were fixed at days 3, 5, 7, 9 or 11 with $3 \%$ paraformaldehyde (PFA/PBS) (Fluka). Histological aspects of the cultures were assessed by Hoffman contrast observations (Nikon microscope). Experiments were repeated three times.

Reverse transcription-polymerase chain reaction (RT-PCR) assay Total RNA was isolated using PureLink Micro-to-Midi Total RNA Purification System (Invitrogen) or High Pure RNA Isolation Kit (Roche). The mRNA encoding MMP-2 or MMP-9 fragment was amplified by a reverse transcription-polymerase chain reaction assay (RT-PCR) using $0,1 \mu \mathrm{g}$ of total RNA as a template, with the sets of previously described primers (Son et al., 2006) and a Titan One Tube RT-PCR Kit (Roche Diagnostic), according to the manufacturer's instructions. Primers for housekeeping gene (Mendler et al., 1998, Zador et al., 1996) glyceraldehyde-3-phosphate dehydrogenase (GAPDH) were:

5'-TTCACCACCATGGAGAAGGC-3' and

5'-CAGGAGACAACCTGGTCCTC-3'. RT-PCR conditions (35 cycles) were as follow: reverse transcription at $50^{\circ} \mathrm{C}$ for $30 \mathrm{~min}$, then $94^{\circ} \mathrm{C}, 30$ $\mathrm{sec} ; 50^{\circ} \mathrm{C}, 45 \mathrm{sec} ; 68^{\circ} \mathrm{C}, 45 \mathrm{sec}$. Obtained cDNA fragments were separated on $2 \%$ Agarose LE gels (Roche Diagnostics). The gels were stained with ethidium bromide and analyzed with Gel Doc 2000 using the Quantity One software (BioRad).

\section{Western blotting analysis}

Analyses were performed for the regenerating muscles and differentiating myoblasts. In vivo regenerating muscle or cells grown in vitro were homogenised in a buffer containing $20 \mathrm{mM}$ Tris- $\mathrm{HCl}, 5 \mathrm{mM}$ EGTA, 
$5 \mathrm{mM}$ EDTA, $150 \mathrm{mM} \mathrm{NaCl}, 0.01 \%$ leupeptin, 0.5mM PMSF, $1 \%$ Triton $\mathrm{X}-100$ (Sigma), $\mathrm{pH}$ 7,5. All operations were performed on ice. Protein concentration was determined as previously described (Bradford, 1976). Samples containing $20 \mu \mathrm{g}$ of proteins were separated by SDS PAGE 10\% gels transferred to PVDF membrane (Roche), and incubated with primary rabbit polyclonal antibody against MMP-9 or MMP2 (Chemicon) at final dilution 1:200 (overnight, $4^{\circ} \mathrm{C}$ ). Subsequently, the blots were incubated with secondary anti-rabbit antibodies conjugated to peroxidase (Santa Cruz Biotechnology) for $1.5 \mathrm{hrs}$, at room temp, at final dilution 1:10000. Chemiluminescence detection was performed with the Lumi-Light Pluse Western Blotting Substrate (Roche) according to the manufacturer's protocol. Blots were documented using $\mathrm{Gel}$ Doc 2000 scanner. Each experiment was performed three times.

\section{Gelatinase extraction}

Extraction of MMP-9 or MMP-2 was performed for the regenerating muscles and cell conditioned medium. Enzymes were extracted as previously described (Zhang and Gottschall, 1997). In brief, the intact and regenerating muscles were homogenized in buffer containing $50 \mathrm{mM}$ Tris- $\mathrm{HCl}, 150 \mathrm{mM} \mathrm{NaCl}, 5 \mathrm{mM} \mathrm{CaCl}_{2}, 0,05 \%$ Brij 35, $1 \%$ Triton X100 (Sigma), pH 7,6 using $5 \mathrm{ml}$ Teflon glass homogenizer. Muscle homogenate or cell conditioned medium $(500 \mu \mathrm{l})$ were incubated for 90 min with $50 \mu \mathrm{l}$ of gelatin-Sepharose 4B (Amersham Bioscience) with constant shaking. Samples were washed with buffer containing $50 \mathrm{mM}$ Tris- $\mathrm{HCl}, 150 \mathrm{mM} \mathrm{NaCl}, 5 \mathrm{mM} \mathrm{CaCl}_{2}, 0,05 \%$ Brij $_{3}$ (Sigma), pH 7,6 and pellets were incubated for 30 min with $150 \mathrm{ml}$ of elution buffer containing $50 \mathrm{mM}$ Tris- $\mathrm{HCl}, 150 \mathrm{mM} \mathrm{NaCl}, 5 \mathrm{mM} \mathrm{CaCl}, 0,05 \%$ Brij $35,10 \%$ DMSO (Sigma) pH 7,6. Gelatinase activity in the samples was measured by standard zymography as described below.

\section{Assay of MMP activity}

Zymography was performed as previously described (Hibbs et al., 1985). In brief, samples were mixed with non-reducing sample buffer containing $62,5 \mathrm{mM}$ Tris $\mathrm{HCl}, 10 \%$ glycerol, $2 \%$ SDS, $0,05 \%$ bromophenol blue (Sigma) and loaded onto 7,5\% SDS-polyacrylamide gels containing $0,1 \%$ gelatin (Sigma). In addition, a sample containing $5 \mathrm{ng}$ of gelatinase zymography standards (Chemicon) was run together with experimental samples on each gel. After electrophoresis, SDS was removed from the gels by washing in $2,5 \%$ Triton $\mathrm{X}-100$ ( $2 \times 20 \mathrm{~min})$. $\mathrm{Next}$, for $48 \mathrm{~h}$, gels were incubated in buffer containing $50 \mathrm{mM}$ Tris- $\mathrm{HCl}$, $5 \mathrm{mM} \mathrm{CaCl}_{2}, 200 \mathrm{mM} \mathrm{NaCl}$ (Sigma), pH 7,5, stained with Coomassie blue (BioRad) and scanned using the Gel Doc 2000. software. Each experiment was performed three times. According to gelatinase zymography standards, MMP-9 was detected as a band at approximately $98 \mathrm{kDa}$ corresponding to MMP-9 proenzyme. MMP-2 was detected as the proenzyme that can be seen at approximately $68 \mathrm{kDa}$. A secondary band of the activated form at approximately $62 \mathrm{kDa}$ was also seen.

\section{Immunolocalization of MMP}

Immunolocalization of MMP-9 or MMP-2 was performed for the regenerating muscles in vivo and differentiating myoblasts in vitro. Myoblasts at different stages of in vitro differentiation were fixed with $3 \%$ PFA/PBS (Fluka) and permeabilized with 0,1\% Triton/PBS (Sigma). The transverse sections of the intact or regenerating muscles $(10 \mu \mathrm{m}$ thick) were obtained using a cryostat (MICROM). Non-specific sites were blocked with $3 \%$ BSA/PBS (Fluka) for $1 \mathrm{~h}$. Next, sections or cells grown in vitro were incubated with primary rabbit polyclonal antibody against MMP-9 or MMP-2 (Santa Cruz Biotechnology) in 3\% BSA/PBS (Fluka) at final dilution of $1: 100$. Then muscle sections were incubated with secondary anti-rabbit antibodies conjugated with FITC fluorochrome (Chemicon) at final dilution 1:200. Cells grown in vitro were incubated with secondary anti-rabbit antibody conjugated with Alexa fluorochrome (Invitrogen) at final dilution 1:200. Myoblasts nuclei were stained with chromomycin (Sigma). The images were recorded in the
LSM 510 confocal system (Zeiss) and analyzed with LSM 5 Rel. 3.2 software.

\section{References}

ALLBROOK, D. (1981). Skeletal muscle regeneration. Muscle Nerve 4: 234-45.

ALLEN, D.L., TEITELBAUM, D.H. and KURACHI, K. (2003). Growth factor stimulation of matrix metalloproteinase expression and myoblast migration and invasion in vitro. Am J Physiol Cell Physio/284: C805-15.

ANDERSON, J.E. (1998). Murray L. Barr Award Lecture. Studies of the dynamics of skeletal muscle regeneration: the mouse came back! Biochem Cel/ Bio/76: 13-26.

BASSAGLIA, Y. and GAUTRON, J. (1995). Fast and slow rat muscles degenerate and regenerate differently after whole crush injury. J Muscle Res Cell Motil16: 420-9.

BRADFORD, M.M. (1976). A rapid and sensitive method for the quantitation of microgram quantities of protein utilizing the principle of protein-dye binding. Anal Biochem 72: 248-54.

CHAMBERS, R.L. and MCDERMOTT, J.C. (1996). Molecular basis of skeletal muscle regeneration. Can J App/ Physio/21: 155-84.

CHARGE, S.B. and RUDNICKI, M.A. (2004). Cellular and molecular regulation of muscle regeneration. Physiol Rev 84: 209-38.

DARR, K.C. and SCHULTZ, E. (1987). Exercise-induced satellite cell activation in growing and mature skeletal muscle. J App/ Physio/63: 1816-21.

DEMESTRE, M., ORTH, M., WELLS, G.M., GEARING, A.J., HUGHES, R.A. and GREGSON, N.A. (2005). Characterization of matrix metalloproteinases in denervated muscle. Neuropathol App/ Neurobio/31: 545-55.

EL FAHIME, E., TORRENTE, Y., CARON, N.J., BRESOLIN, M.D. and TREMBLAY, J.P. (2000). In vivo migration of transplanted myoblasts requires matrix metalloproteinase activity. Exp Cell Res 258: 279-87.

HAAS, T.L., MILKIEWICZ, M., DAVIS, S.J., ZHOU, A.L., EGGINTON, S., BROWN M.D., MADRI, J.A. and HUDLICKA, O. (2000). Matrix metalloproteinase activity is required for activity-induced angiogenesis in rat skeletal muscle. Am JPhysiol Heart Circ Physio/279: H1540-7.

HIBBS, M.S., HASTY, K.A., SEYER, J.M., KANG, A.H. and MAINARDI, C.L. (1985). Biochemical and immunological characterization of the secreted forms of human neutrophil gelatinase. J Biol Chem 260: 2493-500.

HUARD, J., LI, Y. and FU, F.H. (2002). Muscle injuries and repair: current trends in research. $J$ Bone Joint Surg Am 84-A: 822-32.

KALHOVDE, J.M., JERKOVIC, R., SEFLAND, I., CORDONNIER, C., CALABRIA E., SCHIAFFINO, S. and LOMO, T. (2005). «Fast» and "slow» muscle fibres in hindlimb muscles of adult rats regenerate from intrinsically different satellite cells. J Physio/562: 847-57.

KHERIF, S., LAFUMA, C., DEHAUPAS, M., LACHKAR, S., FOURNIER, J.G., VERDIERE-SAHUQUE, M., FARDEAU, M. and ALAMEDDINE, H.S. (1999) Expression of matrix metalloproteinases 2 and 9 in regenerating skeletal muscle: a study in experimentally injured and mdx muscles. Dev Bio/205: 15870.

KIESEIER, B.C., SCHNEIDER, C., CLEMENTS, J.M., GEARING, A.J., GOLD, R., TOYKA, K.V. and HARTUNG, H.P. (2001). Expression of specific matrix metalloproteinases in inflammatory myopathies. Brain 124: 341-51.

KUGLER, A. (1999). Matrix metalloproteinases and their inhibitors. Anticancer Res 19: $1589-92$

LAGORD, C., SOULET, L., BONAVAUD, S., BASSAGLIA, Y., REY, C., BARLOVATZ MEIMON, G., GAUTRON, J. and MARTELLY, I. (1998). Differential myogenicity of satellite cells isolated from extensor digitorum longus (EDL) and soleus rat muscles revealed in vitro. Cell Tissue Res 291: 455-68.

LEWIS, M.P., TIPPETT, H.L., SINANAN, A.C., MORGAN, M.J. and HUNT, N.P. (2000). Gelatinase-B (matrix metalloproteinase-9; MMP-9) secretion is involved in the migratory phase of human and murine muscle cell cultures. $J$ Muscle Res Cell Motil21: 223-33.

MAURO, A. (1961). Satellite cell of skeletal muscle fibers. J Biophys Biochem Cytol 9: 493-5.

MCCAWLEY, L.J. and MATRISIAN, L.M. (2001). Matrix metalloproteinases: they're not just for matrix anymore! Curr Opin Cel/ Bio/13: 534-40. 
MENDLER, L., ZADOR, E., DUX, L. and WUYTACK, F. (1998). mRNA levels of myogenic regulatory factors in rat slow and fast muscles regenerating from notexin-induced necrosis. Neuromuscul Disord 8: 533-41.

MORACZEWSKI, J., PIEKARSKA, E., ZIMOWSKA, M. and SOBOLEWSKA, M. (1996). Activity of mu- and $\mathrm{m}$-calpain in regenerating fast and slow twitch skeletal muscles. Acta Biochim Po/43: 693-700.

NONAKA, I., TAKAGI, A., ISHIURA, S., NAKASE, H. and SUGITA, H. (1983). Pathophysiology of muscle fiber necrosis induced by bupivacaine hydrochloride (Marcaine). Acta Neuropathol (Berl)60: 167-74.

OHTAKE, Y., TOJO, H. and SEIKI, M. (2006). Multifunctional roles of MT1-MMP in myofiber formation and morphostatic maintenance of skeletal muscle. $J \mathrm{Cell} S \mathrm{Sc}$ 119: 3822-32.

PARTRIDGE, T. (2006). Disciplining the stem cell into myogenesis. NEng/JMed354: 1844-5.

RAVANTI, L. and KAHARI, V.M. (2000). Matrix metalloproteinases in wound repair (review). Int J Mol Med6: 391-407.

SCHOSER, B.G., BLOTTNER, D. and STUERENBURG, H.J. (2002). Matrix metalloproteinases in inflammatory myopathies: enhanced immunoreactivity near atrophic myofibers. Acta Neuro/ Scand 105: 309-13.

SCHULTZ, E. (1989). Satellite cell behavior during skeletal muscle growth and regeneration. Med Sci Sports Exerc 21: S181-6.

SON, B.R., MARQUEZ-CURTIS, L.A., KUCIA, M., WYSOCZYNSKI, M., TURNER, A.R., RATAJCZAK, J., RATAJCZAK, M.Z. and JANOWSKA-WIECZOREK, A. (2006). Migration of bone marrow and cord blood mesenchymal stem cells in vitro is regulated by stromal-derived factor-1-CXCR4 and hepatocyte growth factor-C- met axes and involves matrix metalloproteinases. Stem Cells 24: 1254-64.

TEIXEIRA, C.F., ZAMUNER, S.R., ZULIANI, J.P., FERNANDES, C.M., CRUZHOFLING, M.A., FERNANDES, I., CHAVES, F. and GUTIERREZ, J.M. (2003). Neutrophils do not contribute to local tissue damage, but play a key role in skeletal muscle regeneration, in mice injected with Bothrops asper snake venom. Muscle Nerve 28: 449-59.

TIDBALL, J.G. (2005). Inflammatory processes in muscle injury and repair. $A m J$ Physiol Regul Integr Comp Physio/288: R345-53.

TSURUDA, T., COSTELLO-BOERRIGTER, L.C. and BURNETT, J.C., JR. (2004). Matrix metalloproteinases: pathways of induction by bioactive molecules. Heart Fail Rev 9: 53-61.

WERB, Z. and CHIN, J.R. (1998). Extracellular matrix remodeling during morphogenesis. Ann N Y Acad Sci857: 110-8.

ZADOR, E., MENDLER, L., VER HEYEN, M., DUX, L. and WUYTACK, F. (1996). Changes in mRNA levels of the sarcoplasmic/endoplasmic-reticulum $\mathrm{Ca}(2+)$ ATPase isoforms in the rat soleus muscle regenerating from notexin-induced necrosis. Biochem J320: 107-13.

ZHANG, J.W. and GOTTSCHALL, P.E. (1997). Zymographic measurement of gelatinase activity in brain tissue after detergent extraction and affinity-support purification. JNeurosci Methods 76: 15-20.

ZIMOWSKA, M., SZCZEPANKOWSKA, D., STREMINSKA, W., PAPY, D., TOURNAIRE, M.C., GAUTRON, J., BARRITAULT, D., MORACZEWSKI, J. and MARTELLY, I. (2001). Heparan sulfate mimetics modulate calpain activity during rat Soleus muscle regeneration. J Cell Physio/188: 178-87. 


\section{Related, previously published Int. J. Dev. Biol. articles}

See our recent Special Issue Developmental Biology in Poland edited by Kloc, Maleszewski and Tarkowski at: http://www.ijdb.ehu.es/web/contents.php?vol=52\&issue=2-3

See our Special Issue Mammalian Reproduction \& Development in honor of Anne McLaren and edited by Brigid Hogan at: http://www.ijdb.ehu.es/web/contents.php?vol=45\&issue=3

Soluble membrane-type 3 matrix metalloprioteinase causes changes in gene expression and increased gelatinase activity during Xenopus laevis development

Logan A. Walsh, Colin A. Cooper and Sashko Damjanovski

Int. J. Dev. Biol. (2007) 51: 389-396

The extracellular matrix in development and regeneration. An interview with Elizabeth D. Hay Robert L. Trelstad

Int. J. Dev. Biol. (2004) 48: 687-694

Matrix metalloproteinases in cancer: from new functions to improved inhibition strategies Alicia R. Folgueras, Alberto M. Pendás, Luis M. Sánchez and Carlos López-Otín Int. J. Dev. Biol. (2004) 48: 411-424

Changes in the proportion and number of Pax(7+ve) and MF20(+ve) myoblasts during chick myogenesis in the head and limb.

Antonio S J Lee, Ming Zhang and Darrell J R Evans

Int. J. Dev. Biol. (2004) 48: 31-38

Myonuclear domain size varies along the lengths of maturing skeletal muscle fibers.

Benjamin W C Rosser, Malcolm S Dean and Everett Bandman

Int. J. Dev. Biol. (2002) 46: 747-754

Limb muscle development.

Bodo Christ and Beate Brand-Saberi

Int. J. Dev. Biol. (2002) 46: 905-914

The role of p53 in vivo during skeletal muscle post-natal development and regeneration: studies in p53 knockout mice.

Jason D White, Collins Rachel, Royce Vermeulen, Marilyn Davies and Miranda D Grounds

Int. J. Dev. Biol. (2002) 46: 577-582

The transforming growth factor-betas: multifaceted regulators of the development and maintenance of skeletal muscles, motoneurons and Schwann cells.

Ian S McLennan and Kyoko Koishi

Int. J. Dev. Biol. (2002) 46: 559-567

M-calpain levels increase during fusion of myoblasts in the mutant muscular dysgenesis (mdg) mouse.

S Joffroy, N Dourdin, J P Delage, P Cottin, J Koenig and J J Brustis

Int. J. Dev. Biol. (2000) 44: 421-428

Multiple stage-dependent roles for histone deacetylases during amphibian embryogenesis: implications for the involvement of extracellular matrix remodeling. S Damjanovski, L M Sachs and Y B Shi

Int. J. Dev. Biol. (2000) 44: 769-776

Role of the extracellular matrix and growth factors in skull morphogenesis and in the pathogenesis of craniosynostosis.

$P$ Carinci, E Becchetti and $M$ Bodo

Int. J. Dev. Biol. (2000) 44: 715-723

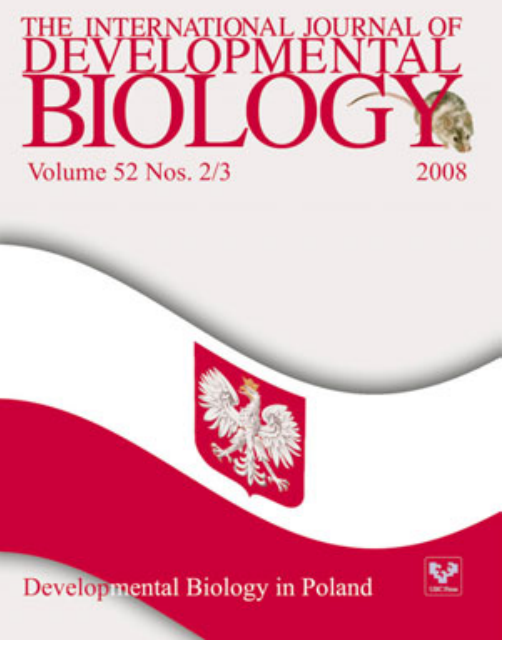

2006 ISI **Impact Factor $=3.577^{\star *}$

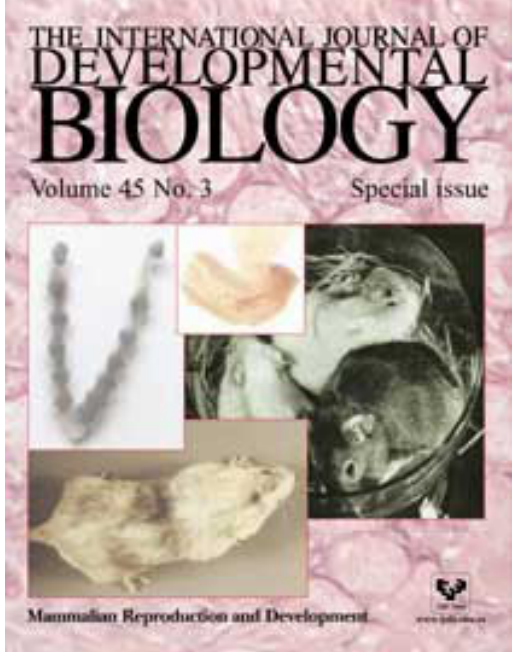

